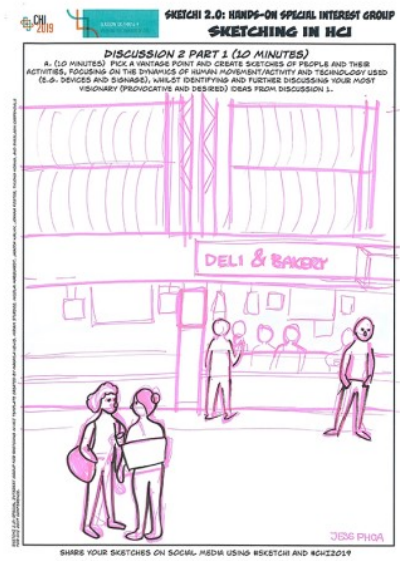

Fig. 1: Sketching atmosphere and participation at $\mathrm{CHI} 2019$ (Jess Phoa) using SketCHI 2.0 handout

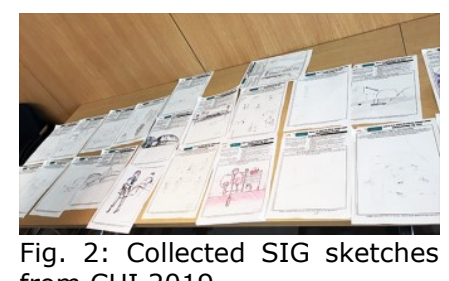
from CHI 2019

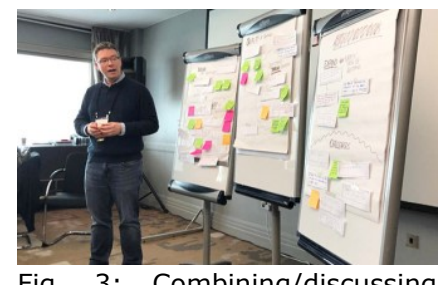

Fig. 3: Combining/discussing attendee thoughts on the role
of sketching in HCI (CHI 2019)

\title{
SketCHI 3.0: Hands-On Special Interest Group on Sketching Education in $\mathrm{HCl}$
}

\begin{tabular}{|c|c|}
\hline Miriam Sturdee & Jess Phoa \\
\hline Lancaster University & Chartbeat \\
\hline United Kingdom & New York, USA \\
\hline m.sturdee@lancaster.ac.uk & jess.phoa@chartbeat.cor \\
\hline Makayla Lewis & Thuong Hoang \\
\hline University of the Arts London & Deakin University \\
\hline United Kingdom & Australia \\
\hline Makayla.Lewis@arts.ac.uk & thuong.hoang@deakin.edı \\
\hline Gonzalo Gabriel Méndez & Sheelagh Carpendale \\
\hline Escuela Superior Politécnica & Simon Fraser University \\
\hline del Litoral & Burnaby \\
\hline $\begin{array}{l}\text { Guayaquil, Ecuador } \\
\text { gmendez@espol.edu.ec }\end{array}$ & $\begin{array}{l}\text { Vancouver, Canada } \\
\text { sheelagh@sfu.ca }\end{array}$ \\
\hline
\end{tabular}

Author Pre-Print - not for distribution

(c) ACM, 2020. This is the author's version of the work. It is posted here by permission of ACM for your personal use. Not for redistribution. The definitive version was published in CHI'20 Extended Abstracts, April 2530, 2020, Honolulu, HI, USA

https://doi.org/10.1145/3334480.3381059

\begin{abstract}
Whilst studying Human-Computer Interaction, students and work-place learners rarely encounter sketching, yet such practice has been shown to improve cognitive processes and increase retention of information. Additionally, it is a valuable method of ideation and communication for both subjective and group-based projects. We propose further integration of sketching practice within $\mathrm{HCI}$ and computer science curricula, both to preserve this valuable skill for use in research and industry, and to widen the perspectives of those working with subjects often seen as grounded in code or logic. SketCHI \#3 will bring together those interested in enhancing student's and colleagues experience in a hands-on meeting of minds and sketching, with the aim to share best practice and knowledge for those interested in expanding our views on education in the field, and to co-create a Sketching in $\mathrm{HCI}$ education plan with a body of knowledge.
\end{abstract}

\section{Author Keywords}

Sketching, Drawing, Visual Thinking, HCI.

\section{CSS Concepts}

- Human-centered computing $\rightarrow$ Visualization $\rightarrow$

Visualization techniques. 


\section{Attendee materials}

A SketCHI 3.0 handout (e.g. Fig. 1) will be given at the beginning of the SIG, however sketching materials will not be provided. It is suggested attendees arrive with light belongings. For example, an A4/A5 hardback spinal sketchbook for additional sketches/to lean on; black drawing pen; 2 colour markers or pencils (a grey to add depth and a colour to highlight important areas). Authors will encourage sketching with pen/markers because mistakes cannot be erased, ensuring attendees keep sketching.

\section{Accessibility}

SketCHI 3.0 will follow the successful format of previous SIG's $[5,7]$ and involve 15 minutes walking around the CHI 2019 conference venue, 25 minutes standing onlocation sketching, and 35 minutes seated in the SIG room. A 10-minute float time has been included to allow for varied attendee walking pace (cont.)

\section{Introduction}

Sketching is a universal tool, and one that has been with us from the earliest days of humanity. We use it to explain, explore - and connect complex themes, as ideation, and as a form of pure artistic creation. This freehand technique is visible in computational form as the stylus and tablet, but the act of making such sketches requires human input, and it is the act of human sketching that we examine within the context of education in HCI. It can be used in higher education (undergraduate and postgraduate), and for peer-topeer learning, where it is often a hitherto unused construct. Once learned, and with continued practice, sketching is often viewed as a useful skill in research and industry settings. Additionally, it is often used in sub-fields and domains of computer science as well as $\mathrm{HCI}$, and in cross-disciplinary settings such as the humanities, psychology and engineering - and further, to elicit ideas and information [11], communicate with teams and stakeholders, to document outcomes and enhance impact via public engagement [3].

Within our schools and higher education systems, the arts appear to suffer at the hands of more "employable" subject areas, but without an arts education we miss out on essential creative thought and communicable skills. Many young adults are put off the visual arts by the notion that they cannot draw [2], or are not good enough [1], and some of the struggle is to recognize and combat these thoughts. After this, the importance of interdisciplinary skills should be imparted, which we propose should happen during undergraduate education, further practiced, and applied in postgraduate education. By integrating sketching in particular to our 'traditional' computing and $\mathrm{HCI}$ education practice, we are teaching something that can be integrated in all areas of learning and for future employment. In its barest forms, sketching is handdrawn imagery for ideation, iteration, interrogation, introspection, and communicable impact [8].

This Special Interest Group on Sketching Education in HCI (SketCHI 3.0) will bring people together to provide evidence and suggest inquiry borne from peer-to-peer learning exercises and experimental work with students in multiple disciplines, to suggest that $\mathrm{HCI}$ (and the wider discipline of computer science) could benefit from embracing the 'humble lines' of hand-drawn imagery. We will discuss methods of integration into existing curricula and the challenges involved whilst conducting hands-on sketching and integrating ourselves into the surroundings of our peers at CHI2020.

\section{SketCHI SIG \# 1}

The first Special Interest Group in Sketching in HCI [5] brought together diverse individuals who all had an interest in the role of sketching in HCI. Whilst sketching our surroundings and the overall atmosphere of the conference, we involved each other in discussions about the significance, pitfalls and benefits of sketching practice in $\mathrm{HCI}$, and further, a working group (SketchingHCI.slack.com) was formed to keep the SIG discussions going after the event. The SIG, alongside a successful course, was written up and published in ACM Interactions as a feature [9] and also produced a featured blog [10].

\section{SketCHI SIG \#2}

The second Sketching in HCI SIG [7] was focused on bringing together practitioners and researchers with a passion for sketching, to discuss and elaborate upon the challenges we face in practicing and celebrating this 


\section{Accessibility (cont.)}

Escalators and lifts will be used if needed during the SIG but we anticipate that only slight elevation may be present. Attendees should be aware that there are seldom chairs or tables when sketching on location, thus comfortable clothing, shoes and a noncumbersome bag, e.g. backpack, is suggested. SketCHI 3.0 is suitable for wheelchair users due to ACM SIGCHI accessibility guidelines; however, it may be unsuitable for people with reduced mobility. To accommodate all attendees, we will provide an alternative schedule (if required) where attendees will remain in the SIG room for facilitated discussions. They will be able to sketch from photos taken by the authors from research environments, or from live streams of concurrent paper presentations. The authors are dedicated to accommodating different access requirements of attendees. skill in the workplace and research outputs. It began with the SIG community sketching the CHI2019 surroundings (Fig. 2) followed by a brainstorming session using flipcharts and post-it notes that offered a way to organize and prioritize the community thoughts (Fig. 3). The SIG concluded with a sketching manifesto task, designed to bring our goals and thoughts together into a coherent message. This Sketching in $\mathrm{HCI}$ manifesto is currently under review for future publication. Additionally, the first, of which we hope many, ACM Interactions featured blog was a published by SketCHI community member:

interactions.acm.org/blog/author/16188/Jess Phoa.

\section{SketCHI SIG \# 3 Goals}

The third Special Interest Group (SketCHI 3.0) revisits the most popular topic from SIG \#1 [5] and SIG \#2 [7]: the education of sketching in HCI. The SketCHI \#1 \& \#2 community has identified that without educating individuals in sketching, and associated skills such as creativity and design thinking [1], we may be limiting our students and continuing learners. Thus, the goal of the third SIG is to co-create an example Sketching in HCI Curricula supported by a body of knowledge to be used in an undergraduate or postgraduate environment (learning population TBD by the SIG community). The intention is to distribute the curricula at a future

SIGCHI conference to enable institutions to add to their learning outcomes and up-skilling.

\section{Presentation \& Schedule}

1. SIG room (10 minutes): Introduction of the SketCHI 3.0 SIG background/goal followed by formation of groups, 4-6 attendees, depending on numbers. Each group will be facilitated by an author. 2. Walk to location 1 and icebreaker (5 minutes):
Attendees, in their groups, will be asked to introduce themselves: name, institution, research interests, and how they use sketching in their HCI research. 3.

Activity \& Discussion \#1 (15 minutes): Attendees will pick a vantage point and create sketches of their surroundings, focusing on details which capture the essence of the conference environment, whilst brainstorming a) 'Who is your target audience for sketching education in HCI?' b) 'What key areas of sketching should be taught? c) Identify when your key ideas should be taught?' 4. Walk to location 2 (5 minutes): attendees will be asked to share their sketches within their group and conclude discussion \#1. 5. Activity \& Discussion 2 (15 minutes): (a) (10 minutes) Attendees will pick a vantage point at location 2 and create sketches of people and their activities, focusing on the dynamics of human movement and technology (e.g. devices and signage), whilst, creating a sketching in HCI tutor persona, using the handouts provided by the authors, that includes background, practice, and skill level. (b) (5 minutes) Attendees will then be asked to identify key resources (industry and academic) to support their curricula (ideas). 6. Walk to SIG room (5 minutes): attendees will be asked to share sketches and post-it note summaries with another group. 7. Co-create SketCHI Education Recommendations (20 minutes): Facilitated by the authors, attendees will share and organize their ideas with the wider SIG and then, using flipchart and post-it notes, co-create a sketching in $\mathrm{HCI}$ education plan that includes topics of interest, activities, and a supportive body of knowledge. 8. SIG room (5 minutes): SketCHI 3.0 will end by recording all the generated data and introducing the sketching in $\mathrm{HCI}$ online community. 


\section{Audience}

SketCHI 3.0 will follow on from the lead authors accepted CHI 2020 courses So You Think You Can't Draw? A Hands-on Introductory Course on Sketching in HCI Techniques and Sketching in HCI: Research Practice \& Publication (Advanced), where attendees will be guided through selected sketching techniques and strategies to produce tangible outputs and engagement with research and industry. SketCHI 3.0 further develops the ideas and community from previous SIGs and focuses on deliberating and delivering practical outputs for use by the wider ACM and SIGCHI community. We aim to attract people from all areas of industry and research to join us in creating an education package that can benefit the whole community. All with an interest are welcome: sketching competence or confidence are not prerequisites.
At SketCHI 3.0 locations, the authors will interact with the groups, sketching, and participating in-group discussions (see SketCHI \#1 [5] and SketCHI \#2 [7]). This SIG takes advantage of the opportunity to utilize the roof terrace to sketch the wider surroundings of the CHI 2020 conference. During the SIG, the authors will ensure the SIG attendees is not in the way of activities of the other conference attendees.

\section{SIG Deliverables}

The organizers will produce a Sketching Education in HCI plan grounded in the SIG discussions to be shared with attendees and the wider HCI community.

Precisely, the plan will be written up and disseminated via a future ACM SIGCHI conference. At the end of SketCHI 3.0, the authors will introduce the 'Sketching in $\mathrm{HCI}^{\prime}$ online community (SketchingHCI.slack.com) on Slack, aims to enable continued discussions,

collaborations, and resource sharing. To join the online 'Sketching in HCI' community attendees will be asked to complete a short Google form: goo.gl/forms/vva3hSSqswax3QuC2.

\section{References}

[1] Sunni Brown. The doodle revolution: Unlock the power to think differently. Portfolio, 2015.

[2] Neil Cohn. Explaining 'I can't draw': Parallels between the structure and development of language and drawing. Human Development 55, no. 4 (2012): 167-192.

[3] Makayla Lewis, Makayla, Miriam Sturdee, Jason Alexander, Jelle Van Dijk, Majken Kirkegård Rasmussen, and Thuong Hoang. SketchingDIS: Hand-drawn sketching in HCI. In Proceedings of the 2017 ACM Conference Companion Publication on Designing Interactive Systems, pp. 356-359. ACM, 2017.
[4] Makayla Lewis, Makayla, Miriam Sturdee, and Nicolai Marquardt. Applied Sketching in HCI: Hands-on Course of Sketching Techniques. In Extended Abstracts of the 2018 CHI Conference on Human Factors in Computing Systems. ACM, 2018.

[5] Makayla Lewis, Miriam Sturdee, Nicolai Marquardt, Thuong Hoang. SketCHI: Hands-On Special Interest Group on Sketching in HCI. In Extended Abstracts of the 2018 CHI Conference on Human Factors in Computing Systems. ACM, 2018.

[6] Makayla Lewis, Miriam Sturdee, and Nicolai Marquardt. Sketching in HCI: Hands-on Course of Sketching Techniques. In Extended Abstracts of the 2019 CHI Conference on Human Factors in Computing Systems. ACM, 2019.

[7] Makayla Lewis, Miriam Sturdee, Jagoda Walny, Nicolai Marquardt, Thuong Hoang, Joanna Foster, and Sheelagh Carpendale. SketCHI 2.0: Hands-On Special Interest Group on Sketching in HCI. In Extended Abstracts of the 2019 CHI Conference on Human Factors in Computing Systems. ACM, 2019.

[8] Miriam Sturdee. Sketching as a support mechanism for the design and development of shape-changing interfaces. PhD diss., Lancaster University, 2018.

[9] Miriam Sturdee, Makayla Lewis, and Nicolai Marquardt. Feeling SketCHI?: the lasting appeal of the drawn image in HCI. interactions 25, no. 6 (2018): 64-69.

[10] Miriam Sturdee, Makayla Lewis, and Nicolai Marquardt. SketchBlog\# 1: the rise and rise of the sketchnote. interactions 25, no. 6 (2018): 6-8.

[11] Miriam Sturdee and Joseph Lindley. Sketching \& Drawing as Future Inquiry in HCI. In Proceedings of the Halfway to the Future Symposium 2019, p. 18. ACM, 2019. 